\title{
Effects of wedge degradation on the magnitude of the Ponzo illusion
}

\author{
ALICE QUANTE LIBET, ROBERT H. POLLACK, and VICTOR J. MALATESTA \\ University of Georgia, Athens, Georgia 30602
}

\begin{abstract}
This research represents an attempt to test empirically three different theoretical explanations for the classic Ponzo illusion: perspective theory, lateral inhibition theory, and assimilationcontrast theory. Under conditions of tachistoscopic presentation $(250 \mathrm{msec}), 24$ male and 24 female undergraduate students were shown the full-wedge Ponzo figure and two degradations, each with either apical or distal line position. Differences in magnitude and direction of illusion among the figures revealed equivocal findings. In accounting for the Ponzo illusion, the results question the adequacy of each theoretical formulation. The weight of the evidence, however, shows greater support for the assimilation-contrast notion.
\end{abstract}

In 1912 Mario Ponzo introduced the illusion that has become the classical Ponzo illusion. Although direct research on the Ponzo illusion is sparse, different explanations have been proposed to account for its occurrence (cf. Over, 1968). The Ponzo figure typically consists of an inducing wedge or angle within which the lengths of two equal lines appear unequal. The line located nearer the apex of the wedge (apical line) appears to be longer than a line located farther away (distal line).

The most popular explanation for this apparent inequality of two lines located within the wedge is based upon the use of perspective cues. If the angle is oriented with its apex at the top of the figure, the appearance is that of railroad tracks converging in the distance. The figure also corresponds to one artistic representation of depth: converging lines. The interpretation of depth results in the overestimation of the apical line in comparison with the distal line, since their relative sizes are judged with respect to their apparent distance from the observer.

Gregory (1963) has criticized perspective theory in explaining the Ponzo illusion, since there is a conflict with the principles of perspective. In a three-dimensional situation, a stimulus closer to the eye appears to be longer than a stimulus of the same size which is placed farther away. In the Ponzo figure it is the line which is apparently farther away that appears to be longer. Thus, the traditional perspective theory is a contradiction of the "laws of perspective" in that it predicts that distant objects appear larger, not smaller, than nearer objects. Gregory (1963) suggested that the misapplication of the constancy principle results in the illusion. He speculated that the compensation for changes in the retinal image which accompanies

Requests for reprints should be addressed to Robert $\mathrm{H}$. Pollack, Department of Psychology, University of Georgia, Athens, Georgia 30602. the perception of depth causes the apparent lengthening of the apical line

Leibowitz, Brislin, Perlmutter, and Hennessy (1969) incorporated both perspective theory and the inappropriate size-constancy ideas in accounting for the Ponzo illusion. They demonstrated that the Ponzo illusion may be enhanced by the addition of perspective cues. In this study, subjects were shown stimuli ranging from an actual three-dimensional scene of railroad tracks, to photographs of three-dimensional scenes containing many perspective cues, to a simple geometric figure of the Ponzo illusion. They found that, as perspective cues were removed, the magnitude of the illusion decreased. However, a $10 \%$ illusion remained when only the geometric figures were shown. In addition, rotation of the geometric figure did not change the magnitude of the illusion, although such manipulation may be presumed to alter depth cues.

Lateral inhibition notions that focus upon retinal processes have been developed (Ganz, 1966; von Békésy, 1967). In this formulation, the peaks of two adjacent contours are shifted away from each other due to the stronger inhibition of the parts of the distribution which lie closest to each other. As a result of this mutual inhibition, the apparent location of the contours is displaced. Hubel and Wiesel (1962) stated that the amount of inhibition depends on the relative spatial location, intensity, and the temporal sequence of the stimuli. Ratliff (1971) modified the lateral inhibition notion somewhat. He stated that the strongest inhibition does not occur immediately adjacent areas of excitation, but instead at those some distance out. The inhibition then decreases gradually as the distance between areas of excitation increases.

Over (1968), in his review of explanations of geometric illusions, pointed out that a problem arises with respect to the distances of neural interaction. If contour separation is an important determinant of the illusion, in some cases the nueral interaction 
must occur only over limited separation, and in others it must operate over greater distances.

Both perspective theory and lateral inhibition were proposed by Kohler and Wallach (1944). They showed subjects standard and variable lines in six positions, with the standard line ranging from an extreme apical location to a distal location just outside of the ends of the wedge. The greatest underestimation of the standard line occurred when the line was located at the ends of the wedge. The greatest overestimation occurred when the line was located near the apex of the wedge. Quina and Pollack (1971) proposed an assimilationcontrast explanation of the Ponzo illusion. According to their formulation, the line located near the apex of the wedge essentially completes a triangle. The triangular configuration results in an apparent lengthening of lines placed near the apex of the wedge. Those lines located farther from the apex appear to shrink due to the contrast of the line length with the spaces between the wedge contours and the ends of the lines. Both assimilation and contrast forces may be operating at the same time, but one will dominate the other. The total illusion in the classical figure is thus conceived to be two separate but summating illusions, with dominant assimilation forces resulting in overestimation of apical lines and dominant contrast forces resulting in underestimation of distal lines.

Pressey (1971) formulated the assimilation notion somewhat differently. He stated that the apical line inside the wedge assimilates with the intersecting oblique lines, resulting in an elongation of the apical line. The line located at the end of the wedge has no lines with which to assimilate; therefore, it appears to be comparatively shorter. Pressey demonstrated that a triangular configuration may have the effect of making a line appear shorter than a line without the converging angle. He found that the converging oblique lines added above the standard line decrease the effect of diverging oblique lines below the standard. Pressey also demonstrated that the illusion may occur without the presence of the full wedge.

Coren (1970) modified the Poggendorff and Mueller-Lyer illusion figures to dotted figures. He found that the classical illusions still occurred, but the magnitude of the illusion was significantly smaller for the dotted figures than for the standard figures. Coren stated that the contour interactions are not necessary for the existence of classical geometric illusions, although the decrement in illusion magnitude found for the dotted figures may indicate that the lateral inhibition contributes to the total illusion. On the other hand, this decrement may be due to "impoverishment of the stimulus and hence the attendant illusion cues" (p. 277). $\mathrm{He}$ concluded that lateral inhibition alone cannot account for the classical geometric illusions.

Over (1968) suggested that the greatest gains in understanding geometric illusions could be made by subjecting some of the current theories to further research. He stated that the best strategy is to determine illusion magnitudes under conditions in which different theories would make different predictions about the illusion magnitude. The present study was designed to follow the strategy suggested by Over (1968) in investigating perspective theory, lateral inhibition theory, and the assimilation-contrast theory proposed by Quina and Pollack (1971) as explanations for the Ponzo illusion.

The present study employed the full-wedge Ponzo figures used by Quina and Pollack (1971) and two figures in which the wedge was degraded (Figure 1).

The positions of the standard line within the full wedge were the positions Quina and Pollack (1971) found to result in the greatest overestimation (line 1) and the greatest underestimation of line length (line 2). These degradations were chosen in order to determine possible differences in magnitude and direction of illusion among the figures that would support or question the adequacy of the three major explanations of the Ponzo illusion.

Perspective theory would predict that, in the fullwedge figure, the apical line should be overestimated to a lesser degree. Since the wedge is a perspective cue, degrading the wedge should reduce the cue. Therefore, the magnitude of illusion should be greater for the full-wedge than for the degraded-wedge figures. In the dots-only condition, in which there are no perspective cues, the illusion should disappear.
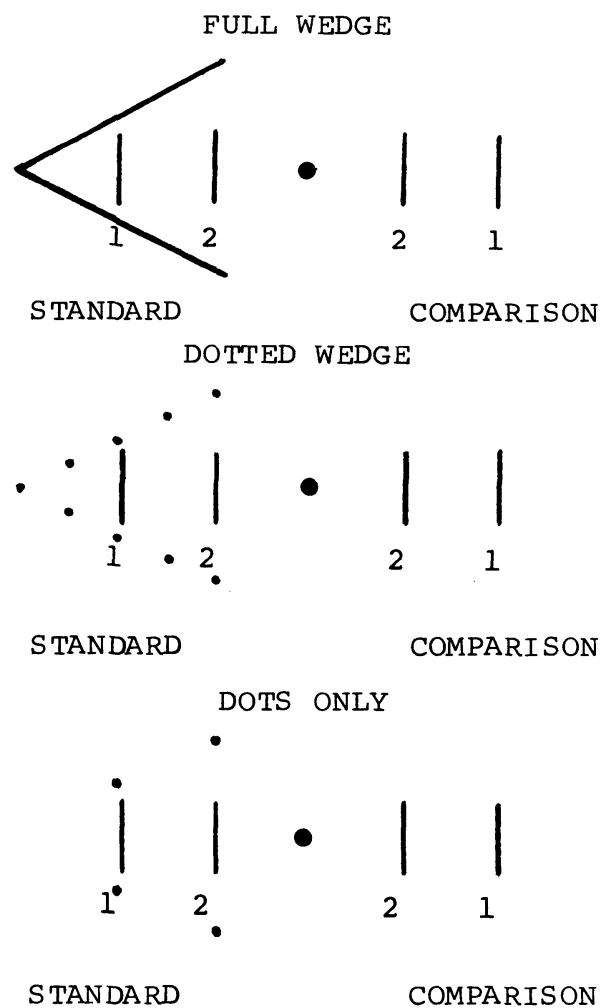

Figure 1. The Ponzo figure and degradations of the figure used in the present study. 
Satiation and lateral inhibition would predict an underestimation of both apical and distal lines. The distal line would expect to shrink more than the apical line, since the ends of the distal lines would lie in the area of greater satiation. The magnitude of this negative illusion should be greater for the full-wedge figures than for the degraded figures, since a reduction in contour would reduce lateral inhibition and satiation effects.

Assimilation-contrast theory would predict that the apical line would be overestimated and the distal line would be underestimated. In addition, the magnitude of the illusion for the apical line should be greater for the full-wedge than for the degraded-wedge figures. Degrading the wedge reduces its triangularity and, therefore, assimilation effects. The magnitudes of illusion for the full-wedge and degraded-wedge figures should not differ from each other when the line is in the distal position. In this case, the illusion depends only on the comparison of line length with the spaces between the wedge contours and the ends of the line. Degrading the wedge should not reduce contrast effects.

\section{METHOD}

\section{Subjects}

Subjects were 24 male and 24 female white undergraduate students, aged 18 to 24 years, who were enrolled in introductory psychology courses. Each subject had an uncorrected visual acuity of $20 / 25$ or better, as measured by Snellen's Distance Vision Test Letters.

\section{Apparatus and Stimuli}

Stimuli were presented in a three-channel tachistoscope (Scientific Prototype, Model GB). Illumination was constant for all channels at $16.076 \mathrm{~mL}$, as measured by a Macbeth illuminometer.

Stimulus figures were drawn in black India ink on white $5 \times 7$ in. cards. No figure subtended a visual angle of more than $3 \mathrm{deg} 15 \mathrm{sec}$. The full-wedge Ponzo figure consisted of a 60-deg inducing angle whose apex was located at $38.4 \mathrm{~mm}$ from a central fixation point $2 \mathrm{~mm}$ in diameter. The ends of this angle were located $9.57 \mathrm{~mm}$ from the fixation point. An 11-mm standard line was shown in one of two positions inside the inducing wedge. In the apical position, the standard line was located $24 \mathrm{~mm}$ from the fixation point. In the distal position, the standard line was located $9.57 \mathrm{~mm}$ from the fixation point. A comparison line was located equidistant from the fixation point on the opposite side. The length of the comparison line varied, in $.5-\mathrm{mm}$ steps, from 7.5 to $14.5 \mathrm{~mm}$. The position of the wedge at the right or left of the fixation point was randomized. All lines were $.5 \mathrm{~mm}$ wide.

The first degradation of the illusion figure was a dotted wedge (Figure 1) composed of nine .5-mm-diam dots. One dot was the vertex and four dots $7.5 \mathrm{~mm}$ apart composed each virtual contour of the wedge. The standard line in the apical position had dots located $3 \mathrm{~mm}$ from its ends, and the standard line in the distal position had dots $11.5 \mathrm{~mm}$ from its ends.

The second degradation of the Ponzo wedge (the dots-only figure) consisted of the standard line colinear with $.5-\mathrm{mm}$ dots near each end (Figure 2). The distance between the dots and the ends of the standard line was $3 \mathrm{~mm}$ for the apical position and $11.5 \mathrm{~mm}$ for the distal position.

A control condition consisted of the standard line with no surrounding figure. The standard line was compared with a line located equidistant from the fixation point on the op- posite side. The location of the apical and distal lines corresponded to the apical and distal positions described previously. The comparison line varied in length from 10 to $12 \mathrm{~mm}$, in 5-mm steps.

\section{Procedure}

Subjects were dark-adapted for $5 \mathrm{~min}$; during this time they were read a standard set of instructions and received three practice trials. Subjects were asked whether the comparison line was longer or shorter than the standard line for each figure shown. Each of the stimuli was presented for $250 \mathrm{msec}$ in one channel of the tachistoscope. A blank field displaying only a 2-mm fixation point remained on in the background channel except during stimulus presentations.

Four presentations of the control series were made, followed by six presentations of the series for each of the wedge conditions. The order of the three experimental conditions was randomized across subjects. Four additional presentations of the control series followed the experimental presentations. Each subject viewed the control and experimental series for either the apical or distal line position only. Thus, 12 male and 12 female subjects viewed the stimuli for each line position. A converging method of limits was used in the presentation of each series.

Points of subjective equality (PSEs) were calculated for the control and experimental trials. The mean control PSE was subtracted from the mean of each experimental PSE for each subject to obtain the net magnitude of illusion.

\section{RESULTS}

A 2 by 3 (line position by wedge condition) mixed-model factorial analysis of variance revealed a significant main effect for wedge condition but not for line position $[F(2,92=9.31, p<.001]$. A significant interaction effect was also found $[F(2,92)=16.35, p<.001]$.

Further analysis revealed a negative illusion for both apical and distal line positions, indicating an underestimation of the standard line in all figures (see Figure 2). The Newman-Keuls test indicated that

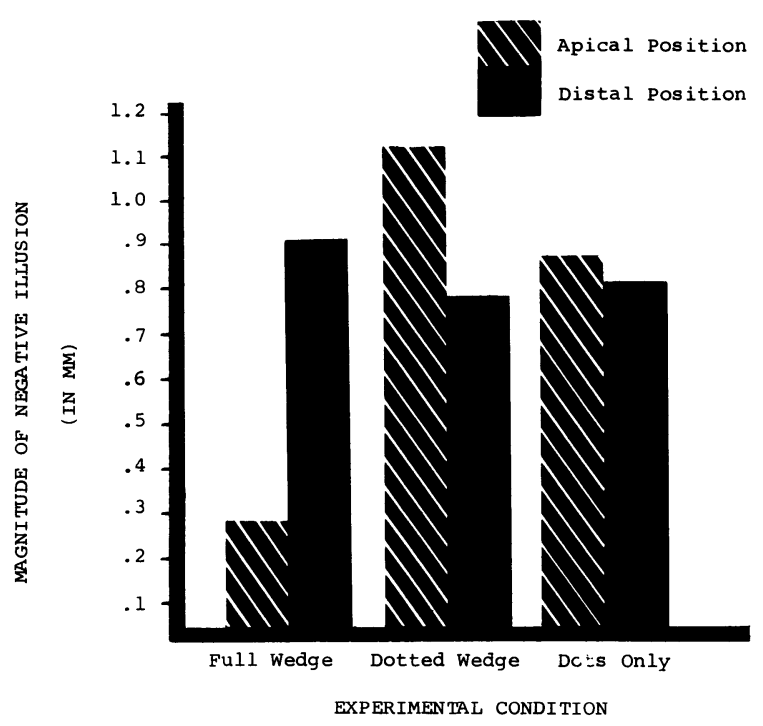

Figure 2. Magnitude of illusion for experimental conditions at each position. 
all illusion magnitudes were significantly different from zero $(p<.05)$. Significant differences were found among illusion magnitudes with the line in the apical position $(\mathrm{p}<.01)$, but none was found among illusion magnitudes for figures with the line in the distal position $(\mathrm{p}<.05)$.

The Newman-Keuls test also indicated that the negative value obtained for the full-wedge figure with the line in the apical position was significantly smaller $(p<.01)$ than those values obtained in any other wedge condition for both line positions. The dotted wedge figure with the line in the apical position resulted in a significantly larger negative illusion than did any other condition for both line positions $(p<.01)$. The means obtained from the dots-only conditions for both apical and distal lines did not differ significantly from each other $(p>.05)$.

No significant sex differences were found.

\section{DISCUSSION}

The results of this study do not confirm predictions made by perspective theory. Contrary to the prediction of overestimation of the apical and distal lines in the full-wedge figure, both lines were underestimated. It must be pointed out, however, that the apical line was underestimated significantly less than was the distal line for the full-wedge figure $(p<.001)$. This may be interpreted as partially supporting the perspective theory, since their comparative lengths are in the predicted direction.

According to perspective theory, since the wedge functions as a perspective cue, degrading the wedge would reduce the cue, and, thus, the magnitude of the illusion should be smaller in degraded-wedge figures. This prediction was not confirmed. Degrading the wedge for lines in the distal position did not change the magnitude of the illusion, while degrading the wedge for lines in the apical position increased the illusion. Perspective theory would seem to have difficulty accounting for the negative illusion found in the dots-only condition for both line positions, since in these figures the angle that is presumed to suggest depth is absent. According to perspective theory, no illusion should occur in the dots-only figures.

The prediction of underestimation of both apical and distal lines by the lateral inhibition and satiation theories was confirmed. In addition, the distal line was reduced more than the apical line in the full-wedge figure.

However, the magnitude of the negative illusion did not change with wedge degradations as predicted by these theories. A reduction of contour reduces the magnitude of negative illusion if satiation and lateral inhibition are operating. The significant increase in the illusion magnitude when the wedge was degraded for apical lines poses a serious question for lateral inhibition and satiation theories. The absence of a change in the magnitude of illusion for distal lines further questions adequacy of these theories in explaining the Ponzo illusion.

The assimilation-contrast theory, as formulated by Quina and Pollack (1971), predicts overestimation of the distal line. This prediction was not confirmed. Although the present study failed to replicate the positive illusion for the full-wedge figure with the line in the apical position, the negative illusion found in this study was much smaller than for any other figure. The higher level of illumination used in the present study $(16.076 \mathrm{~mL}$ vs. $4.834 \mathrm{~mL})$ may have made the apical line appear more distinct from the wedge contour. The difference in illumination seems to be an important factor under conditions of rapid exposure.
The notion that the triangular configuration may weaken or override contrast effects is supported by the large negative illusion found for lines in the apical position when the wedge was degraded. This conclusion is further supported by the fact that 8 of 24 subjects gave responses indicating lengthening of the apical line for the full-wedge figure, in contrast to no more than 2 such responses for any other stimulus condition.

The assimilation-contrast notion is further supported by the absence of differences among illusion magnitudes for the three conditions when the line was in the distal position. Assimilation-contrast theory predicts no change in illusion magnitude under these conditions, since the contrast effects are independent of contour manipulations so long as the spaces at the ends of the lines are not changed.

Assimilation-contrast theory seems to have difficulty accounting for the absence of differences in the magnitude of illusion for the apical and distal lines, since it is not clear how the contrast effect operates over different comparisons of space to produce the same illusion magnitude.

No other explanation, however, seems to offer a plausible alternative to the assimilation-contrast theory. That is, no other explanation can predict or account for the large shrinkage obtained for apical lines when the wedge was degraded. In addition, the other major theories cannot predict or account for the absence of differential effects in the degraded-wedge figures with the line in the distal position.

The results of this study indicate that the entire wedge is not necessary for an illusion to occur. If both lines were placed together in the wedge under the three conditions, the apical line would appear longer than the distal line; that is, the classical illusion would occur. With the dotted wedge, a small but significant reversed illusion would be expected. In the dots-only figure, the illusion would probably disappear.

\section{REFERENCES}

Coren, S. Lateral inhibition and geometric illusions. Quarterly Journal of Experimental Psychology, 1970, 22, 274-278.

Fisher, G. H. An experimental and theoretical appraisal of the inappropriate size-depth theories of illusions. British Journal of Psychology, 1968, 59, 373-383.

Ganz, L. Mechanism of figural aftereffects. Psychological Review, 1966, 73, 128-150.

GregORY, R. L. Distortion of visual space as inappropriate constancy scaling. Nature, 1963, 199, 678-680.

Hubel, D. H., \& Wiesel, T. N. Receptive fields, binocular interaction, and functional architecture in the cat's visual cortex. Journal of Physiology, 1962, 160, 106-154.

Kohler, W., \& Wallach, H. Figural aftereffects: An investigation of visual processes. Proceedings of the American Philosophical Society, 1944, 73, 285-290.

Leibowitz, H., Brislin, R., Perlmutter, L., \& Hennessy, R. Ponzo perspective illusion as a manifestation of space perception. Science, 1969, 166, 1174-1176.

Over, R. Explanations of geometric illusions. Psychological Bulletin, 1968, 70, 545-562.

Ponzo, M. Rapports de contraste angulaire et l'appreciation de grandeur des astres a l'horizon. Archives Italiennes de Biologie, 1912, 58, 327-329.

Pressey, A. W. An extension of assimilation theory to illusions of size, area, and direction. Perception \& Psychophysics, 1971, 9, 172-176.

Quina, K., \& Pollack, R. Effects of test line position and age on the magnitude of the Ponzo illusion. Perception \& Psychophysics, 1971, 12, 253-256.

Ratliff, F. Contour and contrast. Proceedings of the American Philosophical Society, 1971, 115, 150-163.

von BÉkÉsy, G. Sensory inhibition. Princeton, N.J: Princeton University Press, 1967.

(Received for publication March 8, 1980.) 